\title{
Plasma metabolite profiles in healthy women differ after intervention with supplemental folic acid $v$. folate-rich foods
}

\author{
Mohammed E. Hefni ${ }^{1,2}$, Cornelia M. Witthöft ${ }^{1}$ and Ali A. Moazzami ${ }^{3}$ \\ ${ }^{1}$ Department of Chemistry and Biomedical Sciences, Faculty of Health and Life Sciences, Linnaeus University, 39182 Kalmar, Sweden \\ ${ }^{2}$ Food Industries Department, Faculty of Agriculture, Mansoura University, 35516, PO Box 46, Mansoura, Egypt \\ ${ }^{3}$ Department of Molecular Sciences, Uppsala BioCenter, Swedish University of Agricultural Sciences, Uppsala, Sweden
}

(Received 26 June 2018 - Final revision received 24 October 2018 - Accepted 1 November 2018)

Journal of Nutritional Science (2018), vol. 7, e32, page 1 of 9

doi:10.1017/jns.2018.22

Abstract

Public health authorities recommend all fertile women to increase their folate intake to $400 \mu \mathrm{g} / \mathrm{d}$ by eating folate-rich foods or by taking a folic acid supplement to protect against neural tube defects. In a previous study it was shown that folate-rich foods improved folate blood status as effectively as folic acid supplementation. The aim of the present study was to investigate, using NMR metabolomics, the effects of an intervention with a synthetic folic acid supplement $v$. native food folate on the profile of plasma metabolites. Healthy women with normal folate status received, in parallel, $500 \mu \mathrm{g} / \mathrm{d}$ synthetic folic acid from a supplement $(n 18), 250 \mu \mathrm{g} / \mathrm{d}$ folate from intervention foods $(n 19)$, or no additional folate $(0 \mu \mathrm{g} / \mathrm{d})$ through a portion of apple juice $(n 20)$. The metabolic profile of plasma was measured using ${ }^{1} \mathrm{H}$-NMR in fasted blood drawn at baseline and after 12 weeks of intervention. Metabolic differences between the groups at baseline and after intervention were assessed using a univariate statistical approach $(P \leq 0 \cdot 001$, Bonferroni-adjusted significance level). At baseline, the groups showed no significant differences in measured metabolite concentrations. After intervention, eight metabolites, of which six (glycine, choline, betaine, formate, histidine and threonine) are related to one-carbon metabolism, were identified as discriminative metabolites. The present study suggests that different folate forms (synthetic $v$. natural) may affect related one-carbon metabolites differently.

Key words: Folic acid: Folate: Dietary interventions: One-carbon metabolism: Metabolomics

Folate is a generic term referring to a class of water-soluble B vitamins that are present in mammalian cells in several interchangeable forms. Folate is essential for the biosynthesis of purines and pyrimidines and for amino acid interconversions $^{(1)}$, and therefore good folate status is essential for normal cell division. Low dietary folate intake is linked to an increased risk of fetal neural tube defects and has been suggested as a possible risk factor for colon cancer and $\operatorname{CVD}^{(2,3)}$. Increasing attention is being devoted to the role of folate in mediating one-carbon metabolism ${ }^{(4,5)}$. Folate-mediated onecarbon metabolism (Fig. 1) refers to a network of interrelated reactions that use folate to transfer one-carbon units ${ }^{(1)}$.
According to Nordic Nutrition Recommendations ${ }^{(6)}$, women of productive age are recommended to increase their folate intake to $400 \mu \mathrm{g} / \mathrm{d}$ from folate-rich foods. Recognition of the importance of adequate folate nutrition, and difficulties of meeting the recommendations entirely from dietary sources, have led to the introduction of mandatory folic acid fortification in countries such as the USA, Canada and Chile. Food fortification has effectively reduced the prevalence of neural tube defects ${ }^{(7,8)}$ and congenital heart disorders ${ }^{(9)}$. However, there are concerns that high folic acid intake through food fortification and use of supplements could negatively affect health ${ }^{(10,11)}$. These concerns

Abbreviations: OPLS-DA, orthogonal partial least-squares discriminant analysis; VIP, variable influence of projection.

* Corresponding author: M. E. Hefni, fax +46 4804460 32, email mohammed.hefni@lnu.se

(C) The Author(s) 2018. This is an Open Access article, distributed under the terms of the Creative Commons Attribution-NonCommercial-ShareAlike licence (http://creativecommons.org/licenses/by-nc-sa/4.0/), which permits non-commercial re-use, distribution, and reproduction in any medium, provided the same Creative Commons licence is included and the original work is properly cited. The written permission of Cambridge University Press must be obtained for commercial re-use. 


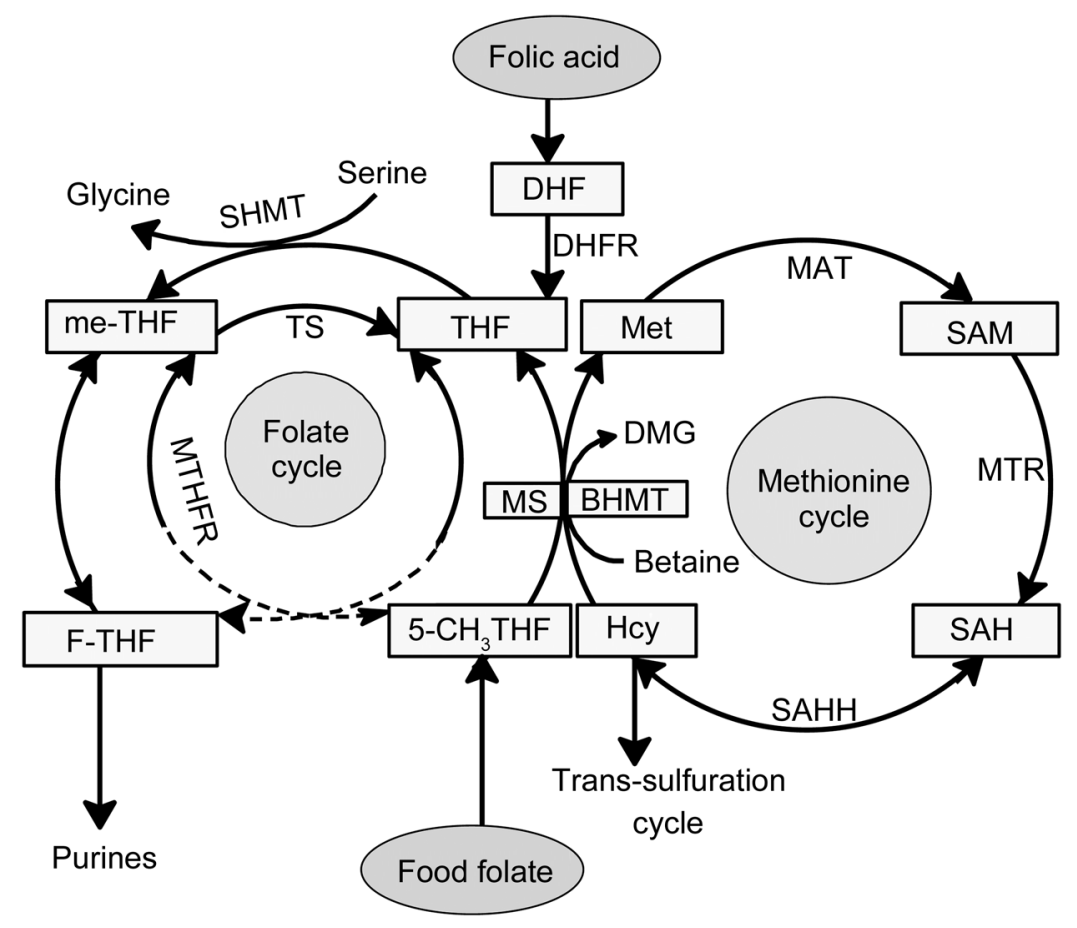

Fig. 1. Folate-mediated one-carbon metabolism. Folic acid has no coenzyme activity and needs to be reduced via dihydrofolate (DHF) to tetrahydrofolate (THF) by dihydrofolate reductase (DHFR). THF is metabolised via serine hydroxymethyl transferase (SHMT) to 5,10-methylene-tetrahydrofolate (me-THF). Serine, by conversion to glycine, donates a one-carbon unit during methylation of THF. Native food folates appear reduced and mainly methylated in the form of 5-methyltetrahydrofolate (5- $\left.\mathrm{CH}_{3} \mathrm{THF}\right)$. An increase in plasma glycine concentration after ingestion of folic acid supplement compared with food folate results in an altered serine:glycine ratio. BHMT, betaine-homocysteine methyltransferase; DMG, dimethylglycine; F-THF, 10-formyltetrahydrofolate; Hcy, homocysteine; MAT, methionine adenyltransferase; Met, methionine; MS, methionine synthase; MTR, methyl transferase; MTHFR, methylenetetrahydrofolate reductase; SAH, S-adenosylhomocysteine; SAHH, S-adenosyl homocysteine hydrolase; SAM, S-adenosylmethionine; TS, thymidylate synthase.

have caused some countries to decide against mandatory folic acid fortification and have prompted investigators to study the effects of folic acid on health.

As previously reported, the 12 -week controlled intervention trial comparing synthetic folic acid $(500 \mu \mathrm{g} / \mathrm{d})$ supplement $v$. natural food folate $(250 \mu \mathrm{g} / \mathrm{d})$ resulted in a similar increase of plasma and erythrocyte folate and a similar decrease of plasma total homocysteine ${ }^{(12)}$. Some metabolic studies, however, have indicated that synthetic folic acid is metabolised differently in the human body from natural food folates ${ }^{(13-15)}$. But these results were based on conventional clinical folate biomarkers, which do not allow to determine downstream metabolic effects. While previous studies have examined the effect of intervention with synthetic folic acid on one-carbon metabolism and related metabolites ${ }^{(16,17)}$, none of these studies compared the metabolic effects of folic acid with natural food folate. Furthermore, in both previous studies pharmaceutical doses $(7.5 \mathrm{mg} / \mathrm{d}$ for 8 weeks and $5 \mathrm{mg} / \mathrm{d}$ for 3 weeks) of folic acid were administered ${ }^{(16,17)}$.

Recent advances in metabolomics using high-throughput analytical methods, e.g. NMR and MS, provide a detailed description of a large number of metabolites present in a biofluid at a specific time. These approaches could be a key tool for in-depth studies on the effects of different folate forms (e.g. natural folate $v$. synthetic folic acid) on various downstream metabolic pathways, important information that is currently lacking.
The aim of the present study was to investigate, by applying an NMR-based metabolomics approach, the effects of an intervention with synthetic folic acid supplement $v$. native food folate on the profile of metabolites in plasma.

\section{Subjects and methods}

\section{Subjects}

A set of plasma samples was obtained from volunteers who participated in a previous intervention study comparing the effects of synthetic folic acid and natural food folate on folate status ${ }^{(12)}$. Nutritional adequacy of participants was indicated by normal folate status (plasma folate $>20 \mathrm{nmol} / 1$, erythrocyte folate $>600 \mathrm{nmol} / \mathrm{l}$, vitamin $\mathrm{B}_{12}>300 \mathrm{pmol} / \mathrm{l}$ and plasma total homocysteine $<12 \mu \mathrm{mol} / \mathrm{l}$ ) (Supplementary Table S1) prior to intervention. Normal biochemical ranges of fasted plasma glucose, Fe status ( $\mathrm{Hb}$, serum ferritin), liver status (aspartate transaminase, alanine transaminase and $\gamma$-glutamyl transferase activity) and lipid profile were also required. Other inclusion criteria were: no history of acute or chronic disease, no use of vitamin or mineral supplements or folic acid-fortified foods, a BMI of between $>18$ and $<30 \mathrm{~kg} / \mathrm{m}^{2}$, no use of any medication interfering with folate metabolism, non-smoker, not consuming a special diet (vegetarian) and no pregnancy, planned conception or lactation. A total of fiftyseven healthy women of reproductive age (19-32 years) met 
the inclusion criteria, were recruited in February 2013 and successfully completed the study. All participants gave written informed consent. The Institutional Review Board of the Faculty of Nursing, Mansoura University, Egypt, reviewed and approved the study protocol. This trial was registered at clinicaltrials.gov as NCT02373033.

\section{Study design}

A randomised, controlled, parallel intervention trial with two active groups and one blind control group was carried out over 12 weeks (March-June 2013) with fifty-seven eligible free-living, apparently healthy women of reproductive age. For an increase in erythrocyte folate concentrations of 50 nmol/1 with $80 \%$ power (two-sided $P<0 \cdot 05)^{(18)}$, fourteen subjects had to complete each intervention diet, as calculated from the standard deviations $(50 \mathrm{nmol} / \mathrm{l})$ of another intervention trial with similar doses ${ }^{(19)}$. Participants were randomly allocated to receive, in addition to their freely chosen diet: (i) folate-rich foods (germinated faba bean stew (200 g), chickpea biscuits $(100 \mathrm{~g})$ and orange juice $(245 \mathrm{ml})$, providing an additional $250 \mu \mathrm{g} / \mathrm{d}$ folate, $n$ 19); (ii) a synthetic folic acid supplement (Arab Co. for Pharmaceuticals \& Medicinal Plants MEPACO, purchased from a local pharmacy in Mansoura, Egypt, providing an additional $500 \mu \mathrm{g}$ folic acid/d, $n$ 18); or (iii) a placebo (apple juice containing $0 \mu \mathrm{g}$ folate, $n$ 20) (Supplementary Table S1). Preparation of the intervention foods is explained elsewhere ${ }^{(12)}$.

\section{Dietary folate and energy intake assessments before and during the study}

To estimate dietary folate and energy intake and to ascertain compliance with the intervention, the subjects were asked to complete a FFQ covering eighty-five items/foods, after screening and after every 4 weeks during the study. As Egypt has no national food composition database for folate, the United States Department of Agriculture (USDA) database was used for estimation of dietary folate intake (http://ndb. nal.usda.gov). For typical Egyptian foods for which no folate data were available in the USDA database, folate data were taken from Hefni $e t a l .{ }^{(20)}$. To measure their compliance, subjects were asked to keep daily records on their consumption of the folic acid supplement, the intervention foods and possible medication or sickness during the dietary intervention.

\section{Folate quantification in the intervention foods}

Folate content and stability in intervention foods were repeatedly quantified in duplicate throughout the trial using HPLC-UV/fluorescence detection ${ }^{(20)}$. Food samples were extracted using tri-enzyme treatment for beans and di-enzyme treatment for bread, juice and cookies. After purification, folates were quantified using reversed-phase-HPLC-UV/ fluorescence detection (Shimadzu LC10) after separation on an Aquasil C18 column $(3 \mu \mathrm{m}, 150 \times 4.6 \mathrm{~mm}$; Thermo Scientific) based on an external multilevel $(n$ 8) calibration curve.

\section{Blood sampling collection and processing}

Blood samples were collected after an overnight fast $(>9 \mathrm{~h})$ in 3.0-ml sodium-heparin vacutainers (Vacutainer ${ }^{\mathbb{B}}$; Zhejiang Gongdong Medical Technology Co., Ltd) at baseline and after 12 weeks of intervention. All samples were immediately placed on ice and centrifuged within $60 \mathrm{~min}$ at $3000 \mathrm{~g}$ for $10 \mathrm{~min}$ at $4^{\circ} \mathrm{C}$. Plasma was separated and stored at $-20^{\circ} \mathrm{C}$ for up to 12 months. Plasma samples were delivered on solid $\mathrm{CO}_{2}$ to the Department of Molecular Sciences, Uppsala, Sweden, and were stored at $-80^{\circ} \mathrm{C}$ until metabolomics analysis.

\section{Sample preparation for NMR analysis}

NMR-based metabolomics analysis of plasma samples was performed according to previously described methods ${ }^{(21,22)}$ after slight modification. In brief, plasma samples were thawed and filtered by ultracentrifuge $\left(10000 \mathrm{~g}, 4^{\circ} \mathrm{C}\right)$ using prewashed (eight consecutive washes with $0.5 \mathrm{ml}$ MQ water at $4000 \mathrm{~g}$ and $36^{\circ} \mathrm{C}$ to remove glycerol) nanosep centrifugal filters with a $3 \mathrm{kDa}$ cutoff (Pall Life Science). Next, $250 \mu \mathrm{l}$ of the filtrate were transferred into a clean microfuge tube and the final volume was made up to $600 \mu \mathrm{l}$ by the addition of $150 \mu \mathrm{l}$ phosphate buffer $(0.4 \mathrm{~mol} / \mathrm{l}, \mathrm{pH} 7 \cdot 0), 45 \mu{ }^{2} \mathrm{H}_{2} \mathrm{O}, 125 \mu \mathrm{l}$ MQ water and $30 \mu \mathrm{l}$ sodium 3-(trimethylsilyl) $\left(2,2,3,3{ }^{2}{ }^{2} \mathrm{H}_{4}\right)$ propionate $(5.8 \mathrm{mmol} / \mathrm{l})$ (Cambridge Isotope Laboratories) as an internal standard. Finally, $560 \mu \mathrm{l}$ of the mixture were transferred to an NMR tube $(5 \mathrm{~mm})$ and capped for analysis.

\section{NMR-based metabolomics analysis}

NMR-based metabolomics analysis of plasma samples was performed on a Bruker spectrometer operating at $600 \mathrm{MHz}$ according to the method described by Moazzami et al. ${ }^{(23)}$. ${ }^{1} \mathrm{H}-\mathrm{NMR}$ spectra of plasma samples were obtained using the zgesgp pulse sequence (Bruker Spectrospin) suppressing water signal at $25^{\circ} \mathrm{C}$ with 128 scans and 65536 data points over a spectral width of $17942.58 \mathrm{~Hz}$. Acquisition time was $1.82 \mathrm{~s}$ and relaxation delay was $4.0 \mathrm{~s}$. The spectral data were processed using Bruker Topspin 1.3 software, Fourier-transformed after multiplication by a line broadening of $0.3 \mathrm{~Hz}$ and referenced to sodium 3-(trimethylsilyl) $\left(2,2,3,3-{ }^{2} \mathrm{H}_{4}\right)$ propionate at $0 \cdot 0$ parts per million. The spectral phase and baseline were corrected manually. Identification of ${ }^{1}$ H-NMR signals was performed primarily using the NMR Suite Library (ChenomX Inc.), the Human Metabolome Database (www.hmdb.ca) and the Biological Magnetic Resonance Data Bank (www.bmrb.wisc.edu).

In all, forty-eight metabolites were identified and their concentrations were calculated using NMR spectral data and NMR Suite Profiler (ChenomX Inc.) by integrating specific spectral regions after accounting for overlapping signals as described previously ${ }^{(23)}$. As in-house quality control, a plasma sample was analysed ( $n$ 30), resulting in CV between 2.8 and $10 \%$ for thirty-five metabolites and CV below $15 \%$ for six metabolites (formate, glutamate, hippurate, sarcosine, threonine and myo-inositol). For further seven metabolites the $\mathrm{CV}$ were higher, namely 2-oxoisocaproate $(17.9 \%)$, 
acetoacetate $(58.2 \%)$, acetone (28.4\%), isopropanol (29.8\%), o-phosphocholine $(21.4 \%)$, propionate $(18.3 \%)$ and trigonelline $(22 \cdot 4 \%)$.

\section{Statistical analysis}

Statistical analysis was performed using both univariate and multivariate approaches. Data were expressed as means and standard deviations.

For univariate data analysis, absolute metabolite concentrations were tested for normality using the Anderson-Darling test, and as a result the data were log-transformed (because the distribution was skewed). Normality of log-transformed data was again tested.

Univariate analysis was used to compare metabolic differences, using one-way ANOVA on log-transformed data between groups at baseline and after the 12-week intervention. A Bonferroni-adjusted significance level of 0.001 was calculated (using the number of variables of forty-eight metabolites) to reduce the chances of obtaining false-positive results (type I error). Paired $t$ tests with a significance level of $P \leq 0.001$ (Bonferroni-adjusted) were also used to analyse differences within the three intervention groups before and after the 12 -week intervention. Univariate analyses were performed using Minitab statistical software, version 17.

For multivariate approach, data were first visualised using principal components analysis to confirm the absence of outliers. For discrimination of the intervention groups and ranking of the metabolites, orthogonal partial least-squares discriminant analysis (OPLS-DA) was used to study the effects of intervention with synthetic folic acid and natural food folate on the metabolic profile to strengthen the findings from univariate analyses. OPLS-DA is a supervised multivariate method which can be used to determine metabolites associated with treatment. Variable influence of projection (VIP) plots were used to determine the discriminative metabolites. Metabolites with VIP $>1$ for which the corresponding jack-knife-based $95 \%$ CI was not close to or not including zero were considered discriminative. The significance of the OPLS-DA model was tested using cross-validated ANOVA with predicted residuals ${ }^{(24)}$. Multivariate analyses were performed using SIMCA-P+ 13.0 software (Umetrics). Results from multivariate analyses are presented in the Supplementary material (Supplementary Table S2 and Supplementary Fig. S1).

\section{Results}

\section{Subject characteristics and folate status}

All fifty-seven subjects completed the study. The baseline characteristics did not differ significantly between the intervention groups (Supplementary Table S1). Subjects were on average 22 years old and had a mean BMI of $24 \mathrm{~kg} / \mathrm{m}^{2}$. Average body weight was maintained throughout the study, with changes within $\pm 5 \%$ of baseline value for all participants. All participants had normal status for folate (plasma and erythrocyte folate and plasma total homocysteine) and vitamin $\mathrm{B}_{12}$ (plasma) at the beginning of the study (Supplementary Table S1). At baseline, there were no significant differences between groups in the concentration of forty-eight plasma metabolites measured using NMR after correction for multiple testing $(P \leq 0 \cdot 001$, Bonferroni-adjusted significance level) (Supplementary Table S1).

The dose of natural folate in the intervention foods $(250 \mu \mathrm{g})$ remained stable during the trial ${ }^{(12)}$.

\section{Metabolic plasma profile after intervention}

After the dietary intervention, twenty-four of forty-eight individual plasma metabolites showed significant differences $(P<$ 0.05) between the different groups (data not shown). However, after Bonferroni correction, only eight metabolites differed significantly $(P \leq 0.001)$, of which six (glycine, choline, betaine, formate, histidine and threonine) are related to one-carbon metabolism (Table 1). Plasma concentrations of glycine, choline, betaine, formate, histidine and threonine before and after the intervention within each intervention group are presented in Fig. 2.

Paired $t$ tests with a significance level of $P \leq 0.001$ (Bonferroni adjusted) were used to compare metabolic differences within the groups (before and after the intervention) and to confirm findings from ANOVA. Folic acid supplementation

Table 1. Concentration ( $\mu \mathrm{mol} / \mathrm{l})$ of plasma metabolites in the control, folate-rich foods and folic acid supplement groups which show significant differences at 12 weeks of intervention*

(Mean values and standard deviations)

\begin{tabular}{|c|c|c|c|c|c|c|c|}
\hline \multirow[b]{2}{*}{ Metabolites $(\mu \mathrm{mol} / \mathrm{l})$} & \multicolumn{2}{|c|}{ Control group } & \multicolumn{2}{|c|}{ Folate-rich foods group } & \multicolumn{2}{|c|}{$\begin{array}{c}\text { Folic acid } \\
\text { supplement group }\end{array}$} & \multirow[b]{2}{*}{$P$} \\
\hline & Mean & SD & Mean & SD & Mean & SD & \\
\hline Glycine & $589^{b}$ & 298 & $357^{c}$ & 191 & $806^{a}$ & 223 & $<0.001$ \\
\hline Choline & $127^{\mathrm{b}}$ & 87 & $60^{c}$ & 61 & $221^{a}$ & 70 & $<0.001$ \\
\hline Betaine & $66^{\mathrm{b}}$ & 18 & $61^{\mathrm{b}}$ & 15 & $85^{a}$ & 18 & 0.001 \\
\hline Formate & $51^{a}$ & 18 & $39^{b}$ & 11 & $57^{a}$ & 13 & 0.001 \\
\hline Histidine & $110^{\mathrm{b}}$ & 28 & $94^{\mathrm{b}}$ & 19 & $132^{\mathrm{a}}$ & 28 & 0.001 \\
\hline Threonine & $178^{a, b}$ & 60 & $138^{\mathrm{b}}$ & 37 & $222^{\mathrm{a}}$ & 66 & 0.001 \\
\hline 2-Oxoiso-caproate & $7^{a}$ & 5 & $3^{b}$ & 2 & $8^{a}$ & 4 & $<0.001$ \\
\hline Propionate & $17^{\mathrm{a}, \mathrm{b}}$ & 8 & $12^{\mathrm{b}}$ & 5 & $21^{a}$ & 9 & 0.001 \\
\hline
\end{tabular}

a,b,c Mean values within a row with unlike superscript letters were significantly different.

* To define significant metabolites, one-way ANOVA on log-transformed data $(P<0.05)$ was used. The Bonferroni-adjusted significance level was defined as $P \leq 0.001$ 

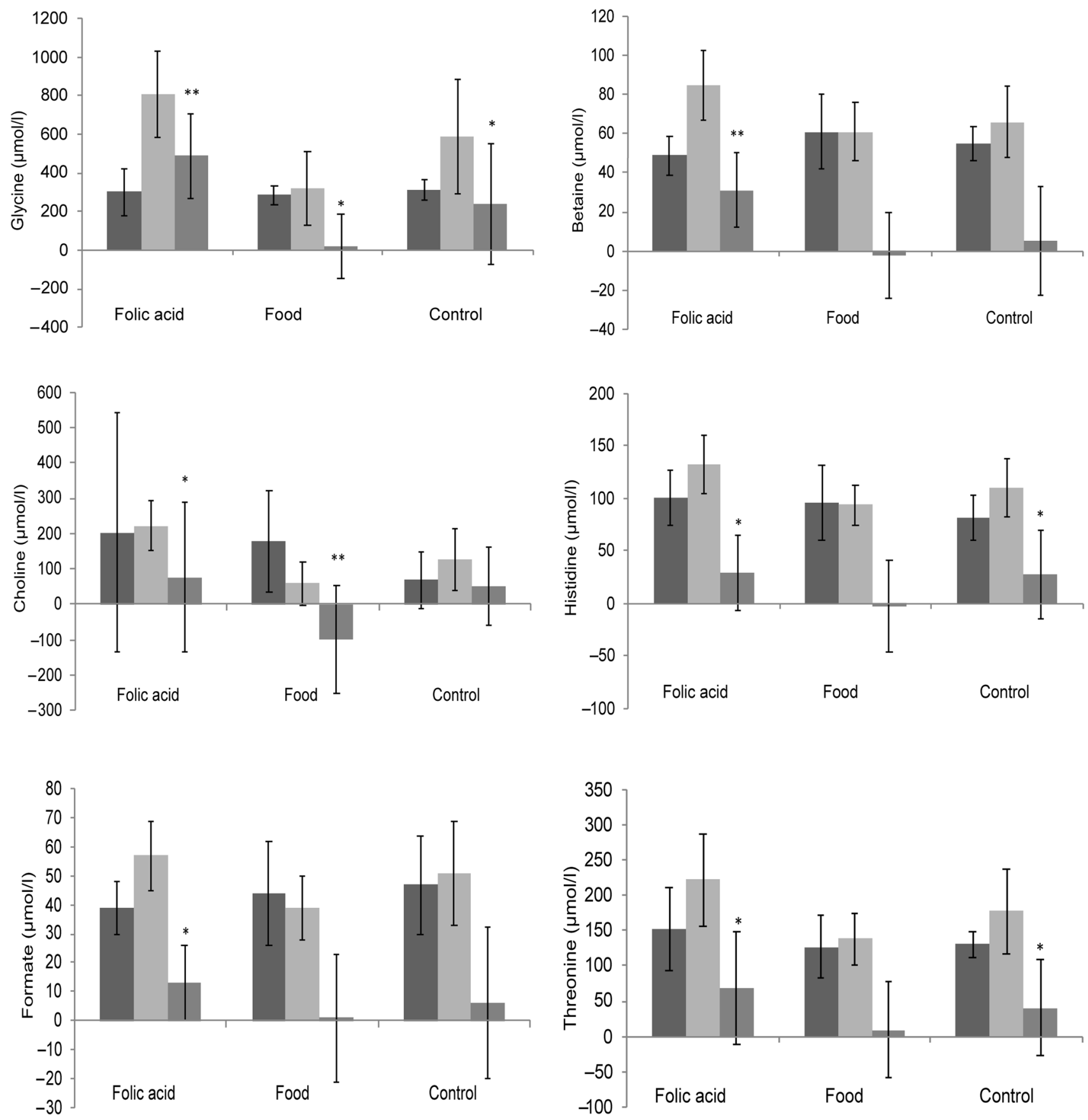

Fig. 2. Plasma concentrations of the six related one-carbon metabolites (glycine, choline, threonine, histidine, betaine and formate) at baseline ( $\square$ ) and after the 12-week intervention $(\square)$, and absolute changes from baseline to 12 weeks $(\square)$. Values are means, with standard deviations represented by vertical bars. Paired $t$ tests with a significance level of $P \leq 0.001$ (Bonferroni-adjusted) were used to analyse differences within the three intervention groups before and after the 12-week intervention. ${ }^{*} P<0.01,{ }^{\star *} P \leq 0.001$.

significantly increased the concentration of ten metabolites (Table 2), of which three (betaine, glycine and 2-oxoisocaproate) were identified by ANOVA. Interestingly, these ten metabolites were not affected in the folate-rich foods group or control group, except for methionine, which was also increased in the control group. In the folate-rich foods group, four metabolites were decreased and one increased $(P \leq 0 \cdot 001)$. Among these metabolites choline and propionate were identified by ANOVA as discriminative metabolites (Table 1).
After intervention, responses for six metabolites varied in the control group (Table 2). In order to correct for these background changes in the metabolic profile of the control group (Table 2), the mean responses of metabolites in both active groups were corrected, by subtraction/addition, for the mean increase/decrease in the control group. For example, the increase in glycine concentration in the control group was subtracted from the absolute increase in the folic acid supplement group. After correction and ANOVA, there was still a significant effect of the folic acid supplement on plasma 
Table 2. Changes $(\mu \mathrm{mol} / \mathrm{l})$ in plasma metabolites within the groups before (baseline) and after the intervention*

(Mean values and standard deviations)

\begin{tabular}{lrrrr}
\hline Metabolites $(\mu \mathrm{mol} / \mathrm{l})$ & & Mean & SD & $P$ (paired $t$ test) \\
\hline Control group & & & & \\
$\quad$ Glutamine & $\uparrow$ & 99 & 127 & 0.001 \\
Methionine & $\uparrow$ & 22 & 21 & $<0.001$ \\
O-Acetyl-carnitine & $\uparrow$ & 4 & 6 & 0.001 \\
Serine & $\uparrow$ & 71 & 117 & $<0.001$ \\
Aspartate & $\downarrow$ & 34 & 41 & $<0.001$ \\
Myo-inositol & $\downarrow$ & 23 & 27 & $<0.001$ \\
Propionate & $\downarrow$ & 11 & 11 & $<0.001$ \\
Folate-rich foods group & & & & \\
Glutamine & $\uparrow$ & 111 & 112 & $<0.001$ \\
Choline & $\downarrow$ & 100 & 153 & $<0.001$ \\
Glutamate & $\downarrow$ & 97 & 117 & $<0.001$ \\
Propionate & $\downarrow$ & 30 & 18 & $<0.001$ \\
Sarcosine & $\downarrow$ & 1 & 1 & $<0.001$ \\
Folic acid supplement group & & & \\
Betaine & $\uparrow$ & 31 & 19 & $<0.001$ \\
Creatinine & $\uparrow$ & 9 & 9 & 0.001 \\
Glycine & $\uparrow$ & 487 & 220 & $<0.001$ \\
Isoleucine & $\uparrow$ & 46 & 35 & $<0.001$ \\
Methionine & $\uparrow$ & 27 & 21 & $<0.001$ \\
Myo-inositol & $\uparrow$ & 31 & 31 & $<0.001$ \\
Leucine & $\uparrow$ & 97 & 62 & $<0.001$ \\
Succinate & $\uparrow$ & 48 & 389 & $<0.001$ \\
2-Oxoiso-caproate & $\uparrow$ & 5 & 4 & 0.001 \\
Valine & $\uparrow$ & 82 & 78 & 0.001 \\
\hline
\end{tabular}

$\uparrow$, Increase; $\downarrow$, decrease.

* The changes are expressed as means of individual data, being calculated as the difference of concentration at 12 weeks minus concentration at baseline. Paired $t$ tests on log-transformed data with a significance level of $P \leq 0.001$ (after Bonferroni adjustment) were used to analyse differences before and after the 12-week intervention.

concentration of glycine $(+37 \% ; P=0.02)$, choline $(+70$ $\% ; P=0.002)$, betaine $(+27 \% ; P=0.008)$ and histidine $(+20 \% ; P=0.03)$. On the other hand, for these metabolites no changes were observed in the folate-rich foods group, except for choline which decreased by $52 \%$ $(P=0 \cdot 002)$.

The increase in plasma glycine concentration after intervention with the folic acid supplement (Fig. 2) resulted in a significant decrease in the serine:glycine ratio $(-30 \%, P<0 \cdot 001$; baseline 0.69 (SD 0.16); after intervention 0.45 (SD 0.13); absolute change -0.32 (SD 0.23$)$ ); this was not observed in either the control or the folate-rich foods group.

In order to use multivariate analyses to strengthen the above results, absence of outliers was confirmed by principal components analysis (data not shown). The metabolic profile of plasma was compared between groups before and after the intervention using OPLS-DA models (data available in the Supplementary material). No discrimination between groups was found before intervention. After intervention, the overall metabolite profile, effectively grouped according to treatment and scores, explained $67 \%$ of the variation (Supplementary Fig. S1). On applying VIP to the profiles after the intervention, glycine was identified as the first discriminative variable (VIP 3.3, $95 \%$ CI 2.9) in the model (Supplementary Fig. S1 and Supplementary Table S2).

\section{Discussion}

The puzzling previous finding that regular consumption of two physiological, but different doses, of two folate vitamers (500 $\mu \mathrm{g}$ synthetic folic acid $v .250 \mu \mathrm{g}$ food folate) results in the same improvement of folate status (based on plasma and erythrocyte folate and plasma total homocysteine) ${ }^{(12)}$ raises questions regarding current assumptions on bioavailability and bioefficacy of synthetic folic acid $v$. endogenous food folates $^{(13)}$. Furthermore, it gives reason to dispute the best means of ensuring that women of reproductive age receive an adequate folate intake. Current recommendations of the Institute of Medicine ${ }^{(25)}$ are based on the theory of a higher bioavailability of supplemental folic acid (85\% when ingested with food) compared with endogenous food folate $(50 \%)$. Data on folate bioavailability are limited ${ }^{(26)}$ and vary from $30 \%{ }^{(27)}$ to $98 \%{ }^{(19)}$. However, findings from several trials have demonstrated that folate-rich foods are as effective as synthetic folic acid in improving folate status ${ }^{(28-31)}$. At the same time it was suggested that differences in the metabolic handling of folic acid and reduced folates might exist ${ }^{(13)}$. However, effects on the profile of metabolites in plasma have not been compared after intervention with synthetic folic acid $v$. food folate.

The present data show distinct metabolic differences after intervention with folic acid supplement $v$. folate-rich foods. Interestingly, six of the eight distinctive metabolites are linked to one-carbon metabolism; the metabolites are glycine, choline, betaine, formate, histidine and threonine (Table 1). This is not surprising, since folate is the corner stone of one-carbon metabolism $^{(1)}$. The individual folate forms enter one-carbon metabolism differently (Fig. 1). Therefore, it is of importance to differentiate between natural reduced folate forms and oxidised synthetic folic acid. Food folate appears largely in the reduced and methylated form (5-methyltetrahydrofolate). Folic acid itself has no coenzyme activity and needs to be enzymically reduced and methylated (Fig. 1). Serine, by conversion to glycine, donates a carbon unit for the methylation of tetrahydrofolate which is the reduced metabolite of dietary folic $\operatorname{acid}^{(32,33)}$. This might explain the increase in plasma glycine concentration after intervention with folic acid supplement compared with the other two groups. Our findings are in agreement with Stam et al. ${ }^{(1)}$, who reported a significant increase in plasma glycine concentration after intervention with folic acid resulting in a $20 \%$ decreased serine:glycine ratio.

Analysis of the metabolic profile in plasma also revealed a significant increase in betaine concentration in the folic acid group compared with the folate-rich foods and control groups. Thus, betaine appeared to be saved in response to folic acid supplementation, as previously reported for adults ${ }^{(34)}$, pregnant women ${ }^{(35)}$ and elderly ${ }^{(36)}$. Findings from the present and previous studies ${ }^{(34-36)}$ indicate that elevated plasma concentrations of betaine can be used as a marker for good folate status. The higher plasma betaine concentration in the folic acid group indicates that synthetic folic acid increases homocysteine remethylation through the methionine synthase pathway (Fig. 1), rather than through the betaine homocysteine 
methyltransferase pathway, thereby saving betaine. Our findings confirm those of others ${ }^{(34,37)}$ which suggest that folic acid supplementation increases plasma betaine. In contrast, betaine supplementation $(6 \mathrm{~g} / \mathrm{d}$, 6 weeks) has no effect on serum folate levels in adults with mildly elevated total homocysteine concentrations ${ }^{(38)}$. This may indicate that the increasing rate of the betaine-homocysteine methyltransferase reaction does not affect the methionine synthase reaction (Fig. 1) ${ }^{(34,38)}$. The increase in plasma choline concentration in the folic acid group compared with the other two groups furthermore confirms that folic acid is saving betaine and thereby choline. This is because choline has been shown to indirectly support homocysteine methylation ${ }^{(39)}$ after being metabolised to betaine.

Another important finding is the lower plasma formate concentration in the folate-rich food group as compared with the folic acid supplement group. In a trial with folatedeficient rats ${ }^{(40)}$, a $44 \%$ higher production rate of formate was observed in animals after repletion with folic acid as compared with deficient rats. Formate is mainly produced in the mitochondria via oxidation of the third carbon of serine ${ }^{(41)}$ and is released into the cytosol using tetrahydrofolate as coenzyme $^{(5)}$ to form 5 -formyltatrahydrofolate for nucleotide synthesis. Hence, the metabolism of formate and the metabolism of folate are interrelated. Folic acid supplementation or fortification was shown to alter the distribution of individual plasma folate forms by increasing the concentration of 5-formyltetrahydrofolate, unmetabolised folic acid and to some extent tetrahydrofolate ${ }^{(42,43)}$. An increased concentration of tetrahydrofolate after folic acid supplementation ${ }^{(42)}$ would explain the elevated concentration of plasma formate in the folic acid supplement group. One could speculate that the increased concentration of formate in the folic acid supplement group is concurrent with an increase of 5-formyltetrahydrofolate, thereby indirectly supporting the above-mentioned findings ${ }^{(42)}$. Unfortunately, we did not measure the distribution of individual folate forms in plasma before or after intervention.

The amino acid histidine is another one-carbon donor which is found to be increased in the folic acid group. Histidine is converted in mammalian tissues via formiminoglutamic acid (FIGLU) in a tetrahydrofolate-dependent pathway to glutamic acid, therefore haematological FIGLU was used as folate status parameter. Folate deficiency has been shown to cause increased histidine excretion into urine, which after folic acid administration returns to its normal level ${ }^{(44)}$. It could be hypothesised that intervention with folic acid results in increased plasma tetrahydrofolate, as reported by Obeid et $a l^{(42)}$, and thereby retains histidine and elevates the plasma concentration.

The amino acid threonine can be converted to glycine, which is used to refuel the folate cycle via the glycine cleavage system $^{(45)}$. However, serine - being converted to glycine - is the main methyl donor in the folate cycle $^{(32)}$. A high plasma glycine concentration after intervention with folic acid could give the impression that rather serine is used as the methyl source instead of other amino acids, thereby sparing threonine.
A limitation of the present study is a lack of control of subjects' protein intake prior to sampling; however, the majority of subjects lived in a university student accommodation and were served the same food in addition to the intervention food. Therefore, it is expected that subjects in all intervention groups had a similar protein intake.

Certain changes in the metabolic profile were observed in the control group after intervention. As it was not likely that this was an effect from diet, other environmental factors, e.g. physical activity, stress or temperature could be causal. Due to lack of control for those factors, we cannot draw any firm conclusions. However, after correction for these background changes, results did not alter.

The intervention foods did not affect habitual food consumption. Most $(>80 \%)$ of the fifty-seven subjects were students living in campus accommodation receiving similar foods all weekdays and habitually consuming conventional canned faba bean stew for dinner. The subjects of the folate-rich foods group were asked to replace their habitual faba bean stew with the germinated faba bean stew and their ordinary breakfast biscuits with the folate-enriched biscuits. Unchanged dietary habits were confirmed by the unaltered BMI (changes $<5 \%$ ) of subjects, which suggests only minor changes in energy intake. Compliance was monitored by daily records of subjects on consumption of the intervention foods and folic acid supplement. Only three subjects reported having failed to take the supplement (during 3-5 d), whereas subjects in the folate-rich foods group reported full compliance. Although subjects lived in a controlled environment, their eating reflects common Egyptian habits.

A limitation of the present study is that the intervention doses for food folate and supplement differed, as the dose of $500 \mu \mathrm{g}$ synthetic folic acid/d was the smallest available in Egypt. On the other hand, it would not have been possible to increase the dose of endogenous food folate without altering dietary habits of subjects in the food group. However, doses could be considered physiological, being within the recommended range of WHO guidelines ${ }^{(46)}$ and Nordic Nutrition Recommendations ${ }^{(6)}$ and both interventions led to similarly improved folate status ${ }^{(12)}$. It is, however, possible that the folate content in the intervention foods was underestimated to a certain extent, as it was quantified by HPLC. The folate content (expressed as the sum of quantified folate forms) determined by HPLC is commonly reported $20-50 \%$ lower than total folate quantified by microbiological assay, which can partly be explained by lack of standards for all folate forms required for HPLC quantification ${ }^{(47)}$.

The main strength of the present study is the use of a metabolomics approach, allowing quantification of a large number of metabolites, i.e. those related to one-carbon metabolism complementing data on traditional folate status parameters.

Summarising, the present findings indicate that synthetic folic acid might alter the balance of one-carbon metabolism and related downstream pathways differently as compared with natural food folate. Results from this initial study show an increase of six distinctive one-carbon metabolites, i.e. glycine, choline, betaine, formate, histidine and threonine, after intervention with supplemental folic acid. This might affect 
the output of one-carbon metabolism, e.g. protein and nucleotide syntheses and whole-body remethylation and transmethylation reactions.

\section{Conclusions}

Findings from the present study suggest that similar improvements in folate status after intervention with natural food folate $v$. folic acid do not reflect differences in underlying metabolic pathways. However, the present results are yet to be considered preliminary due to limitations in the study design. Further studies, using the same doses of folic acid $v$. natural food folate and controlled for protein intake to determine the effects on one-carbon related metabolites, are warranted. The approach of plasma metabolite profiling provides insight into the overall metabolic changes in response to intervention with different folate forms.

\section{Supplementary material}

The supplementary material for this article can be found at https://doi.org/10.1017/jns.2018.22

\section{Acknowledgements}

We are most grateful to all subjects for their participation in the study.

The intervention study was supported by a research grant from Formas-SIDA (Formas Sida Dn 222-2009-1975). Scholarship funding (for M. E. H.) from the Ministry of Higher Education, Egypt, and research funding (for A. A. M.) from the Dr Per Håkansson Foundation are gratefully acknowledged.

M. E. H. participated in planning the trial, coordinated the study, performed the metabolomics analysis and the statistical analysis of data, and drafted the manuscript. C. M. W. was grant holder, designed the study, prepared the ethical application, evaluated the analytical data, and revised the manuscript. A. A. M. supervised the metabolomics analysis, evaluated the statistical analysis and revised the manuscript.

The authors declare no conflict of interest.

\section{References}

1. Stover PJ (2004) Physiology of folate and vitamin $B_{12}$ in health and disease. Nutr Rev 62, S3-S12; discussion S13.

2. Blancquaert D, Storozhenko S, Loizeau K, et al. (2010) Folates and folic acid: from fundamental research toward sustainable health. CRC Crit Rev Plant Sci 29, 14-35.

3. Kennedy DA, Stern SJ, Moretti M, et al. (2011) Folate intake and the risk of colorectal cancer: a systematic review and meta-analysis. Cancer Epidemiol 35, 2-10.

4. Ueland PM, Midttun O, Windelberg A, et al. (2007) Quantitative profiling of folate and one-carbon metabolism in large-scale epidemiological studies by mass spectrometry. Clin Chem Lab Med 45, 1737-1745.

5. Tibbetts AS \& Appling DR (2010) Compartmentalization of mammalian folate-mediated one-carbon metabolism. Annu Rev Nutr 30, $57-81$.
6. Nordic Council of Ministers (2012) Nordic Nutrition Recommendations 2012. Integrating nutrition and physical activity, 5th ed. Nord 2014:002. Copenhagen: Nordic Council of Ministers. http://norden.divaportal.org/smash/get/diva2:704251/FULLTEXT01.pdf (accessed November 2018).

7. De Wals P, Tairou F, Van Allen MI, et al. (2007) Reduction in neural-tube defects after folic acid fortification in Canada. New Engl J Med 357, 135-142.

8. Boulet SL, Yang Q, Mai C, et al. (2008) Trends in the postfortification prevalence of spina bifida and anencephaly in the United States. Birth Defects Res A Clin Mol Teratol 82, 527-532.

9. Ionescu-Ittu R, Marelli AJ, Mackie AS, et al. (2009) Prevalence of severe congenital heart disease after folic acid fortification of grain products: time trend analysis in Quebec, Canada. BMJ 338, b1673.

10. Smith AD, Kim Y-I \& Refsum H (2008) Is folic acid good for everyone? Am J Clin Nutr 87, 517-533.

11. Crider KS, Bailey LB \& Berry RJ (2011) Folic acid food fortification - its history, effect, concerns, and future directions. Nutrients 3, 370-384.

12. Hefni M, Shalaby M, Mohamed M, et al. (2016) Effect of a 12-week dietary intervention with folic acid or folate-enhanced foods on folate status in healthy Egyptian women. FNS 7, 1339-1351.

13. Wright AJA, Finglas PM, Dainty JR, et al. (2005) Differential kinetic behavior and distribution for pteroylglutamic acid and reduced folates: a revised hypothesis of the primary site of PteGlu metabolism in humans. J Nutr 135, 619-623.

14. Sweeney MR, McPartlin J \& Scott J (2007) Folic acid fortification and public health: report on threshold doses above which unmetabolised folic acid appear in serum. BMC Public Health 22, 41.

15. Sweeney MR, Staines A, Daly L, et al. (2009) Persistent circulating unmetabolised folic acid in a setting of liberal voluntary folic acid fortification. Implications for further mandatory fortification? BMC Public Health 18, 295.

16. Villa P, Perri C, Suriano R, et al. (2005) L-Folic acid supplementation in healthy postmenopausal women: effect on homocysteine and glycolipid metabolism. J Clin Endocrinol Metab 90, 4622-4629.

17. Stam F, Smulders YM, van Guldener C, et al. (2005) Folic acid treatment increases homocysteine remethylation and methionine transmethylation in healthy subjects. Clin Sci (Lond) 108, 449-456.

18. Altman DG (1991) Sample size. In Practical Statistics for Medical Research, 1st ed., pp. 455-460. London: Chapman \& Hall.

19. Brouwer IA, van Dusseldorp M, West CE, et al. (1999) Dietary folate from vegetables and citrus fruit decreases plasma homocysteine concentrations in humans in a dietary controlled trial. J Nutr 129, $1135-1139$.

20. Hefni M, Öhrvik V, Tabekha M, et al. (2010) Folate content in foods commonly consumed in Egypt. Food Chem 121, 540-545.

21. Tiziani S, Emwas AH, Lodi A, et al. (2008) Optimized metabolite extraction from blood serum for ${ }^{1} \mathrm{H}$ nuclear magnetic resonance spectroscopy. Anal Biochem 377, 16-23.

22. Moazzami A, Bondia-Pons I, Hanhineva K, et al. (2012) Metabolomics reveals the metabolic shifts following an intervention with rye bread in postmenopausal women - a randomized control trial. Nutr J 11, 88.

23. Moazzami A, Shrestha A, Morrison DA, et al. (2014) Metabolomics reveals differences in postprandial responses to breads and fasting metabolic characteristics associated with postprandial insulin demand in postmenopausal women. J Nutr 144, 807-814.

24. Eriksson L, Trygg J \& Wold S (2008) CV-ANOVA for significance testing of PLS and OPLS ${ }^{\circledR}$ models. J Chemom 22, 594-600.

25. Institute of Medicine (US) Standing Committee on the Scientific Evaluation of Dietary Reference Intakes and its Panel on Folate, Other B Vitamins, and Choline (1998) Dietary Reference Intake; Thiamine, Riboflavin, Niacin, Vitamin $B_{6}$, Folate, Vitamin $B_{12}$, Pantothenic Acid, Biotin, and Choline. Washington, DC: National Academies Press. 
26. Ohrvik VE \& Witthoft CM (2011) Human folate bioavailability. Nutrients 3, 475-490.

27. Hannon-Fletcher MP, Armstrong NC, Scott JM, et al. (2004) Determining bioavailability of food folates in a controlled intervention study. Am J Clin Nutr 80, 911-918.

28. Pinto X, Vilaseca MA, Balcells S, et al. (2005) A folate-rich diet is as effective as folic acid from supplements in decreasing plasma homocysteine concentrations. Int J Med Sci 2, 58-63.

29. Winkels RM, Brouwer IA, Siebelink E, et al. (2007) Bioavailability of food folates is $80 \%$ of that of folic acid. Am J Clin Nutr $\mathbf{8 5}$, 465-473.

30. Fenech M, Noakes M, Clifton P, et al. (2005) Aleurone flour increases red-cell folate and lowers plasma homocyst(e)ine substantially in man. Br J Nutr $\mathbf{9 3}, 353-360$.

31. Öhrvik VE, Olsson JC, Sundberg BE, et al. (2009) Effect of 2 pieces of nutritional advice on folate status in Swedish women: a randomized controlled trial. Am J Clin Nutr 89, 1053-1058.

32. Davis SR, Stacpoole PW, Williamson J, et al. (2004) Tracer-derived total and folate-dependent homocysteine remethylation and synthesis rates in humans indicate that serine is the main one-carbon donor. Am J Physiol Endocrinol Metab 286, E272-E279.

33. Cook RJ (2000) Defining the steps of the folate one-carbon shuffle and homocysteine metabolism. Am J Clin Nutr 72, 1419-1420.

34. Melse-Boonstra A, Holm PLI, Ueland PM, et al. (2005) Betaine concentration as a determinant of fasting total homocysteine concentrations and the effect of folic acid supplementation on betaine concentrations. Am J Clin Nur 81, 1378-1382.

35. Fernàndez-Roig $\mathrm{S}$, Cavallé-Busquets $\mathrm{P}$, Fernandez-Ballart JD, et al. (2013) Low folate status enhances pregnancy changes in plasma betaine and dimethylglycine concentrations and the association between betaine and homocysteine. Am J Clin Nutr 97, 1252-1259.

36. Eussen SJPM, Ueland PM, Clarke R, et al. (2007) The association of betaine, homocysteine and related metabolites with cognitive function in Dutch elderly people. Br J Nutr 98, 960-968.
37. Holm PI, Bleie Ø, Ueland PM, et al. (2004) Betaine as a determinant of postmethionine load total plasma homocysteine before and after B-vitamin supplementation. Arterioscler Thromb Vasc Biol 24, 301-307.

38. Steenge GR, Verhoef P \& Katan MB (2003) Betaine supplementation lowers plasma homocysteine in healthy men and women. J Nutr 133, 1291-1295.

39. Wallace JMW, McCormack JM, McNulty H, et al. (2012) Choline supplementation and measures of choline and betaine status: a randomised, controlled trial in postmenopausal women. Br J Nutr 108, 1264-1271.

40. Morrow GP, MacMillan L, Lamarre SG, et al. (2015) In vivo kinetics of formate metabolism in folate-deficient and folate-replete rats. J Biol Chem 290, 2244-2250.

41. Garcia-Martinez LF \& Appling DR (1993) Characterization of the folate-dependent mitochondrial oxidation of carbon 3 of serine. Biochemistry 32, 4671-4676.

42. Obeid R, Kasoha M, Kirsch SH, et al. (2010) Concentrations of unmetabolized folic acid and primary folate forms in pregnant women at delivery and in umbilical cord blood. Am J Clin Nutr 92, 1416-1422.

43. Pfeiffer CM, Sternberg MR, Fazili Z, et al. (2015) Folate status and concentrations of serum folate forms in the US population: National Health and Nutrition Examination Survey 2011-2. Br J Nutr 113, 1965-1977.

44. Cooperman JM \& Lopez R (2002) The role of histidine in the anemia of folate deficiency. Exp Biol Med (Maywood) 227, 998-1000.

45. Amelio I, Cutruzzolá F, Antonov A, et al. (2014) Serine and glycine metabolism in cancer. Trends Biochem Sci 39, 191-198.

46. World Health Organization (2016) Daily Iron and Folic Acid Supplementation During Pregnancy. http://www.who.int/elena/ titles/daily_iron_pregnancy/en/ (accessed July 2017).

47. Konings EJ, Roomans HH, Dorant E, et al. (2001) Folate intake of the Dutch population according to newly established liquid chromatography data for foods. Am J Clin Nutr 73, 765-776. 